\title{
Rechercher des limites écologiques dans une végétation
}

\author{
J. César ${ }^{1} \mathrm{Ph}$. Daget ${ }^{2}$
}

\section{Mots-clés}

Mesure - Végétation - Méthode - Côte d'Ivoire.

\section{Résumé}

La différence entre deux listes floristiques peut être mesurée par la distance relative de Hamming, c'est-à-dire par le nombre d'espèces qui n'existent que dans un seul des deux relevés rapporté au nombre total d'espèces de l'ensemble des deux listes. L'examen de la variation de cette distance dans une suite de relevés contigus permet de reconnaître des limites écologiques dans le tapis végétal. Son application à un transect de savane en Côte d'Ivoire est discutée.

\section{INTRODUCTION}

Un des écueils le plus souvent rencontré en écologie de terrain est la reconnaissance des limites entre les communautés végétales, qu'elles traduisent des différences de milieux, l'influence d'un arbre, un effet côtier ou un effet de lisière. Ce problème est souvent négligé par les études de phytosociologie ou d'écologie structurelle qui sont plus orientées sur la caractérisation des milieux que sur celle de leurs limites ; l'écologie des paysages étudie davantage la forme des limites que leur nature... Il existe pourtant des méthodes pratiques pour résoudre ces problèmes $(3,4,5)$. L'une d'elles est d'une remarquable simplicité qui la rend apte à un large usage dans les régions où l'équipement matériel est encore insuffisant. Elle est reprise ici et la mise en œuvre est montrée sur un exemple concret de Côte d'Ivoire.

\section{METHODE}

La méthode la plus simple pour aboutir au résultat cherché a été proposée par Godron $(4,5)$, puis présentée et développée par Gauthier et Godron (3). Elle est fondée sur l'application de la distance de Hamming entre deux relevés consécutifs et sa variation le long d'un transect de relevés consécutifs.

En utilisant les conventions usuelles d'écriture (7) :

$\mathrm{a}=$ nombre d'espèces du relevé 1 ,

$\mathrm{b}=$ nombre d'espèces du relevé 2 ,

$\mathrm{c}=$ nombre d'espèces communes aux relevés 1 et 2 ,

la distance de Hamming D entre les relevés 1 et 2 s'écrit :

$\mathrm{D}=\mathrm{a}+\mathrm{b}-2 \mathrm{c}$

1. IDESSA/CIRAD, BP 1465, Bouaké 01,Côte d'Ivoire

2. CIRAD-EMVT/CNRS, Campus international de Baillarguet, BP 5035, 34032 Montpellier Cedex 1, France
En d'autres termes, la mesure de cette distance est égale au nombre d'espèces qui n'existent que dans un seul des deux relevés.

L'expérience montre que le rapport entre la distance effective et la distance maximale Dm (correspondant au cas où les deux relevés consécutifs n'ont aucune espèce en commun) est une mesure plus précise, surtout lorsque le nombre d'espèces par relevé varie de manière notable; elle correspond à :

$$
M^{*}=\frac{D}{D m}=\frac{a+b-2 c}{a+b}=1-\frac{2 c}{a+b}
$$

Dans la dernière partie de cette écriture, on reconnaît (7) le coefficient de communauté de Sörensen S :

$$
S=\frac{2 c}{a+b}
$$

Par conséquent, la distance $\mathrm{M}^{*}$ entre deux relevés est le complément à 1 de ce coefficient :

$$
M^{*}=1-\mathrm{S}
$$

Pratiquement, pour dégager les limites écologiques cherchées, il suffit d'analyser la végétation par un transect constitué d'une suite de $\mathrm{n}$ relevés contigus alignés, que ces relevés soient des segments ou des quadrats rectangulaires $(1,4)$.

Les distances $M^{*}(i, i+1)$ entre le relevé de rang $i$ et le suivant sont mesurées par le nombre d'espèces qui n'existent que dans un seul de ces relevés divisé par le nombre total d'espèces. Elles constituent une séquence de n-1 valeurs.

Il y a une limite écologique lorsqu'apparait une valeur $\mathrm{M}^{*}$ (i) significativement plus élevée que les valeurs voisines. Pour identifier les valeurs significativement plus élevées qu'elles ne le seraient dans une distribution aléatoire, il suffit de comparer leur 
écart à la médiane à l'écart absolu médian (ce dernier étant la médiane des écarts à la médiane) (8) et de prendre comme significatives les valeurs de $\mathrm{M}^{*}$ qui vérifient :

$$
\frac{(\mathrm{M}-\operatorname{med} \mathrm{M})}{\operatorname{med}(\mathrm{M}-\operatorname{med} \mathrm{M})}>\mathrm{L}
$$

avec $\mathrm{L}=5$ pour les écarts hautement significatifs (au seuil de 1 p. 100) et $\mathrm{L}=3$ pour les écarts significatifs (au seuil de $5 \mathrm{p} .100$ ).

\section{APPLICATION}

A titre d'exemple, prenons le premier transect étudié par César (2) en Côte d'Ivoire. Il comporte 20 quadrats consécutifs analysés successivement aux mois de mars et de septembre et, par conséquent, 19 intervalles et 19 distances.

Le tableau I rassemble les éléments du calcul des distances $\mathrm{M}^{*}$; en mars la médiane est de 0,17 et l'écart absolu médian de 0,08, ce qui donne, par application de la formule précédente, des valeurs seuils de 0,41 et 0,57 :

$$
M^{*}=3 \times 0,08+0,17=0,41
$$

En septembre, avec une médiane de 0,33 et un écart absolu médian de 0,08 , les seuils sont de 0,57 et 0,73 . Les figures 1 et 2 montrent la variation spatiale de la distance relative entre relevés voisins.

Les limites majeures, statistiquement significatives au seuil 5 p. 100, sont :

- en mars, entre 13 et 14,14 et 15 , puis 10 et 11 ;

- en septembre, entre 15 et 16 ; des limites moins importantes, nettes mais non significatives, apparaissent ;

- en mars, entre 15 et 16 ;

- en septembre, entre 10 et 11 , et 14 et 15 ; et enfin des limites accessoires peuvent encore être perçues ;

- en septembre, entre 2 et 3,9 et 10 , et 13 et 14 ; les trois lignes du haut de la figure 3 réunissent ces conclusions.

La comparaison avec l'état de la surface du sol montre une zonation mésologique parallèle correspondant au passage du transect sur un affleurement granitique (figure 3, les trois lignes du bas).

La méthode utilisée ne permet pas de porter de jugement sur la nature de la végétation entre deux limites ni de comparer entre elles les régions délimitées. L'examen du tableau de données initial montre cependant une zonation de trois types de végétation : A B C B A dont l'analyse sortirait du cadre de ce travail.

\section{- CONCLUSION}

Dans certaines études de végétation, les limites entre les communautés sont très faciles à dégager. Pour ces situations, relativement exceptionnelles, une technique d'analyse particulière n'est pas nécessaire. Par contre, la méthode de la distance relative de Hamming permet de dégager les limites des communautés végétales dans les cas très fréquents où elles ne sont pas évidentes, souvent parce qu'elles sont masquées par la répartition uniforme

\section{Tableau I}

Nombre d'espèce par quadrat et distance de $\mathrm{H}$ amming entre quadrats dans le transect $A$ de César

\begin{tabular}{|c|c|c|c|c|c|c|}
\hline \multirow[t]{2}{*}{$\mathbf{Q}$} & \multicolumn{3}{|c|}{ Mars } & \multicolumn{3}{|c|}{ Septembre } \\
\hline & $\mathbf{N}$ & D & M* & $\mathbf{N}$ & D & M* \\
\hline \multirow[t]{2}{*}{1} & 11 & & & 11 & & \\
\hline & & 2 & 0,09 & & 7 & 0,30 \\
\hline \multirow[t]{2}{*}{2} & 11 & & & 12 & & \\
\hline & & 3 & 0,14 & & 8 & 0,40 \\
\hline \multirow[t]{2}{*}{3} & 10 & & & 8 & & \\
\hline & & 3 & 0,17 & & 4 & 0,25 \\
\hline \multirow[t]{2}{*}{4} & 8 & & & 8 & & \\
\hline & & 4 & 0,27 & & 5 & 0,33 \\
\hline \multirow[t]{2}{*}{5} & 7 & & & 7 & & \\
\hline & & 2 & 0,15 & & 3 & 0,23 \\
\hline \multirow[t]{2}{*}{6} & 6 & & & 6 & & \\
\hline & & 2 & 0,17 & & 4 & 0,33 \\
\hline \multirow[t]{2}{*}{7} & 6 & & & 6 & & \\
\hline & & 0 & 0 & & 5 & 0,33 \\
\hline \multirow[t]{2}{*}{8} & 6 & & & 9 & & \\
\hline & & 0 & 0 & & 5 & 0,28 \\
\hline \multirow[t]{2}{*}{9} & 6 & & & 9 & & \\
\hline & & 2 & 0,20 & & 7 & 0,41 \\
\hline \multirow[t]{2}{*}{10} & 4 & & & 8 & & \\
\hline & & 3 & 0,60 & & 8 & 0,57 \\
\hline \multirow[t]{2}{*}{11} & 1 & & & 6 & & \\
\hline & & 0 & 0 & & 3 & 0,33 \\
\hline \multirow[t]{2}{*}{12} & 1 & & & 3 & & \\
\hline & & 0 & 0 & & 2 & 0,33 \\
\hline \multirow[t]{2}{*}{13} & 1 & & & 3 & & \\
\hline & & 1 & 1,00 & & 2 & 0,50 \\
\hline \multirow[t]{2}{*}{14} & 0 & & & 1 & & \\
\hline & & 2 & 1,00 & & 4 & 0,66 \\
\hline \multirow[t]{2}{*}{15} & 2 & & & 5 & & \\
\hline & & 4 & 0,50 & & 13 & 0,76 \\
\hline \multirow[t]{2}{*}{16} & 6 & & & 12 & & \\
\hline & & 3 & 0,23 & & 7 & 0,30 \\
\hline \multirow[t]{2}{*}{17} & 7 & & & 11 & & \\
\hline & & 4 & 0,25 & & 5 & 0,23 \\
\hline \multirow[t]{2}{*}{18} & 9 & & & 11 & & \\
\hline & & 4 & 0,20 & & 3 & 0,12 \\
\hline \multirow[t]{2}{*}{19} & 11 & & & 14 & & \\
\hline & & 3 & 0,13 & & 6 & 0,21 \\
\hline 20 & 12 & & & 14 & & \\
\hline
\end{tabular}

(Thèse doct., U niv. Paris VI, France, 1992).

$\mathrm{Q}$ : numéro du quadrat ; $\mathrm{N}$ : nombre d'espèce du quadrat ; $\mathrm{D}$ : distance de Hamming en valeur absolue $; \mathrm{M}^{*}$ : distance de Hamming en valeur relative 


\section{Distance relative}

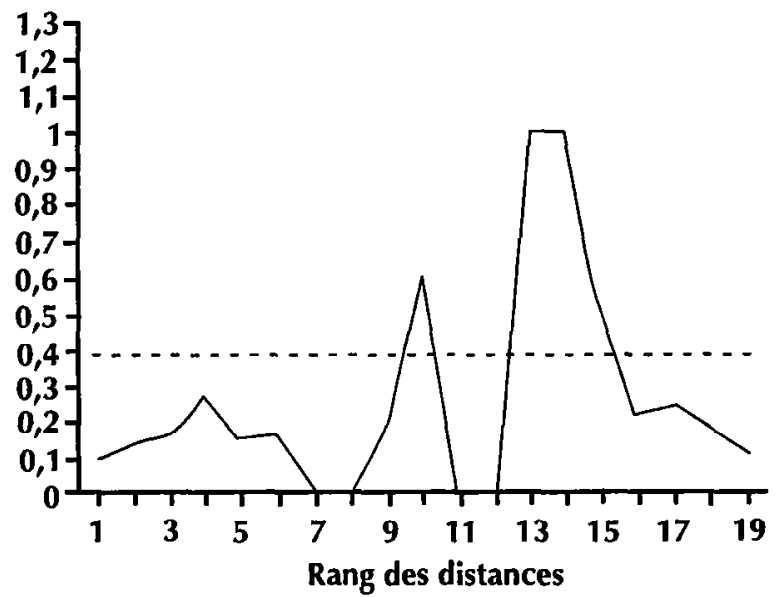

Figure 1 : variation spatiale de la distance relative de Hamming dans le transect $A$ de César (Thèse doct., Univ. Paris VI, France, 1992) au mois de mars.

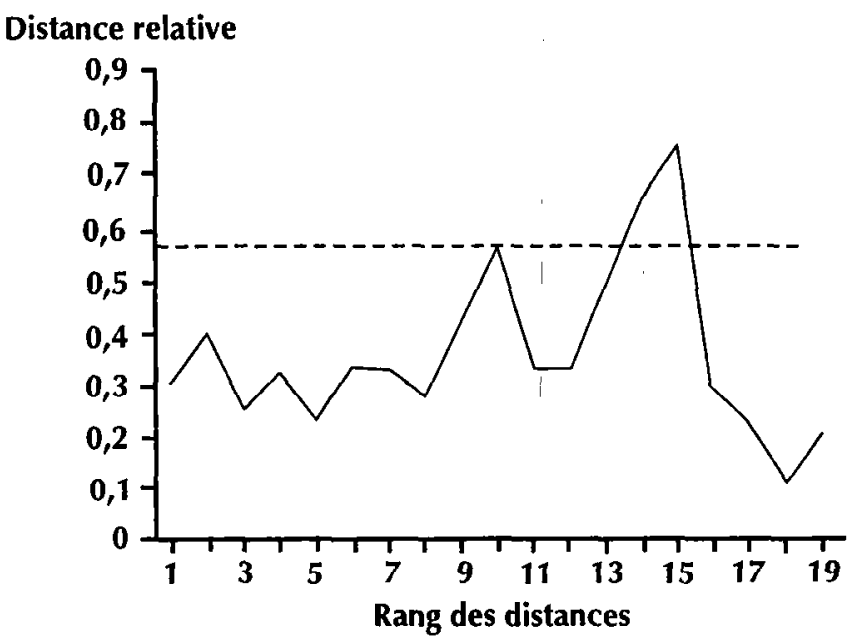

Figure 2 : variation spatiale de la distance relative de Hamming dans le transect $A$ de César (Thèse doct., Univ. Paris VI, France, 1992) au mois de septembre.
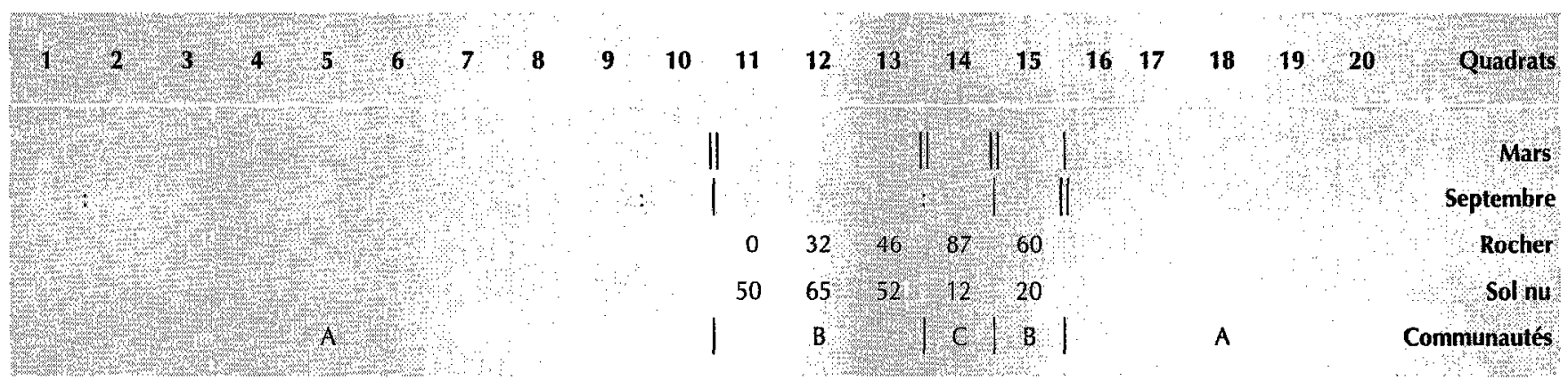

Figure 3 : les limites écologiques dans le transect A de César (Thèse doct., Univ. Paris VI, France, 1992).

d'une espèce dominante. Elle permet, en particulier, de délimiter la zone d'influence d'un arbre sur la végétation herbacée sous-jacente. Toutefois, elle suppose que le passage d'une communauté à sa voisine est relativement rapide à l'échelle de l'étude ; en effet, si ce passage se fait par un continuum progressif largement étalé, cette méthode ne fera rien ressortir de significatif. Par ailleurs, elle ne peut faire ressortir la limite entre deux communautés qui se différencient l'une de l'autre par les recouvrements des espèces plus que par leur liste. Bref, pour que cette méthode puisse faire ressortir une limite, il faut ... qu'il y ait une limite ! Ceci reconnu, la méthode présentée apparaît simple, rapide et performante.

Toutefois, elle reste un peu sommaire parce qu'elle n'utilise qu'une partie de l'information disponible. D'autres méthodes existent, plus sensibles et plus nuancées $(3,5,6)$, mais leur mise en ceuvre est aussi plus délicate, ce qui les rend moins généralisables.

\section{BIBLIOGRAPHIE}

1. DAGET Ph., POISSONET J., 1991. Prairies permanentes et pâturages. Méthodes d'étude. Montpellier, France, Inst. Bot., $390 \mathrm{p}$.
2. CESAR J., 1992. La production biologique des savanes de Côte d'Ivoire et son utilisation par l'homme. Biomasse, valeur pastorale et production fourragère. Maisons-Alfort, France, CIRAD-IEMVT, $672 \mathrm{p}$. (Thèse doct., Univ. Paris VI, France)

3. GAUTHIER B., GODRON M., 1976. La recherche de limites ou de coupures optimale : application à un relevé phytosociologique. Natur. Can., $103: 203-214$.

4. GOUKON M., 1966. Application de la théorie de l'information à l'étude de l'homogénéité et de la structure de la végétation. Oecol. Plant., 1 : 187-197.

5. GODRON M., 1971. Essai sur une approche probabiliste de l'écologie des végétaux. Thèse doct. Etat, Univ. Sci. Tech. Languedoc, Montpellier, France, $247 \mathrm{p}$.

6. GODRON M., BACOU A.M., 1975. Sur les limites optimales séparant deux parties d'une biocénose hétérogène. Ann. Univ. Abidjan, E 7 : 317-324.

7. GOUNOT M., CALLEJA M., 1962. Etude statistique d'une pelouse à Brachypodium ramosum. IV. Coefficients de communauté et homogénéité. Bull. Serv. Carte phytogéogr., série B, 7 : 181-200.

8. SPRENT P., 1992. Pratique des statistiques non paramétriques. Paris, France, INRA, $294 \mathrm{p}$.

Reçu le 30.1 .97 , accepté le 4.8 .97 


\section{Summary}

César J., Daget Ph. Looking for ecological limits in a vegetation cover

The difference between two floristic lists can be measured with Hamming's relative distance, i.e. the number of species that are present in only one of the two relevés is divided by the total number of species in both lists together. Examining distance variations along a series of contiguous quadrats helps find ecological limits in the vegetation cover. The application to a savanna transect in Côte d'Ivoire is discussed.

Key words: Measurement - Vegetation - M ethod - Côte d'Ivoire.

\section{Resumen}

César J., Daget Ph. Búsqueda de los límites ecológicos en la vegetación

La diferencia entre dos muestras florísticos, puede medirse mediante ladistancia relativa de $\mathrm{Hamming}$, es decir por relación entre la cantidad de especies que existen unicamente en una de estas dos muestras, con la cantidad total de especies de las dos muestras juntas. El examen de la variación de esta distancia en una secuencia de muestras contiguas, permite el reconocimiento de los límites ecológicos en la cubierta vegetal. Aplicación a un transecto de la sabana en Costa de Marfil.

Palabras clave: Medición - Vegetación - Método - Costa de Marfil. 